\title{
3D Face Recognition Based on Facial Shape Indexes with Dynamic Programming
}

\author{
Hwanjong Song, Ukil Yang, Sangyoun Lee, and Kwanghoon Sohn* \\ Biometrics Engineering Research Center, \\ Dept. of Electrical \& Electronic Eng., Yonsei University, \\ 134 Shinchon-dong, Seodaemun-gu, Seoul, 120-749, Korea \\ \{ultrarex, starb612\}@diml.yonsei.ac.kr, \\ \{syleee, khsohn\}@yonsei.ac.kr
}

\begin{abstract}
This paper describes a 3D face recognition method using facial shape indexes. Given an unknown range image, we extract invariant facial features based on the facial geometry. We estimate the 3D head pose using the proposed error compensated SVD method. For face recognition method, we define and extract facial shape indexes based on facial curvature characteristics and perform dynamic programming. Experimental results show that the proposed method is capable of determining the angle of faces accurately over a wide range of poses. In addition, $96.8 \%$ face recognition rate has been achieved based on the proposed method with 300 individuals with seven different poses.
\end{abstract}

\section{Introduction}

Over the past few decades, face recognition technologies have made great progress with 2D images, which have played an important role in many applications such as identification, crowd surveillance and access control [1-2]. Although most of the face recognition researches have shown reasonable performance, there are still many unsolved problems in applications with variable environments such as those involving pose, illumination and expression changes. With the development of 3D acquisition system, face recognition based on 3D information is attracting in order to solve problems of using 2D images. A few 3D face recognition approaches have been reported on face recognition using 3D data acquired by 3D sensors [3-5] and stereo-based systems [6]. Especially, most works mentioned above exploited a range image. The advantages of range images are the explicit representation of 3D shape, invariance under change of illumination.

In this paper, we concentrate on the face recognition system using two different 3D sensors. For our system, we utilize the structured light approach for acquiring range data as a probe image and 3D full laser scanned faces for stored images. Fig. 1 briefly presents the whole process of the proposed method. The remainder of this paper is organized as follows: Section 2 describes the representation of $3 \mathrm{D}$ faces for the probe

* Corresponding author. 


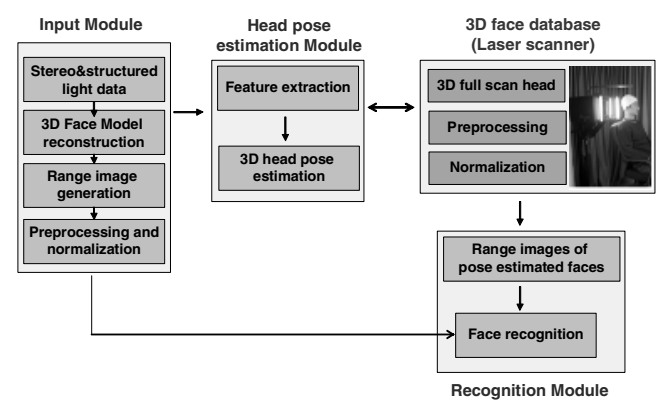

Fig. 1. The block diagram of the proposed method

and store images and describes the extraction of 3D facial feature points. Section 3 introduces an EC-SVD. In section 4, face recognition method is described. In section 5 , test performance is analyzed to explain the efficiency of the proposed algorithm. Finally, section 6 concludes by suggesting future directions.

\section{Representation of 3D Faces}

We acquire a 3D face model from the Genex 3D FaceCam ${ }^{\circledR}$ which is a structured light system in a controlled background. Noise filtering is performed for eliminating the background by some toolkit and we have used the same filter on all images. The orthogonal projection, the range mapping, and projecting uniformly to pixel locations in the image plane are performed with a 3D face model and we generate the range image of the acquired face model. Since the generated range image has some holes to fill due to overlapped or missing the discrete mesh, we use the bilinear interpolation technique.

3D face data is recorded with the Cyberware ${ }^{T M}$ Model 3030PS/RGB highly realistic laser scanner with both shape and texture data. For each 3D face, the scans represent face shapes in cylindrical coordinates relative to a vertical axis centered with respect to the head. In angular steps, angle covers $230^{\circ}$, which means that we scan from the left ear to the right ear. All the faces that we consider are in normalized face space and they are located based on the original face data in the limited range of $[-\sigma, \sigma],[-\varepsilon, \varepsilon],[0, Z]$ for the $\mathrm{X}, \mathrm{Y}$, and $\mathrm{Z}$ axis.

We extract feature points using 3D geometric information. To find the nose peak point (NPP), we select the region from the maximal depth to the depth value lower by three which is empirically found. We calculate the center of gravity of that selected region and treat as an initial NPP. Then we calculate the variances of the horizontal and vertical profiles. We find the points where the minimal variance of the horizontal profiles and the maximal variance of the vertical profiles. We can vertically and almost symmetrically divide the face using the YZ plane which includes the NPP and Y axis, and obtains the face dividing curvature. On the face center curve, we extract facial feature points using curvature characteristics. We finally select six points, which are a minimum point of the nose ridge, the left and right inner eye corner points, a NPP and two nose base points. 


\section{3D Head Pose Estimation}

We describe a 3D head pose estimation algorithm by using 3D facial features. We use them for calculating the initial head pose of the input face based on the Singular Value Decomposition (SVD) method [7]. We utilized EC-SVD to compensate for the rest of the errors which had not yet been recovered from the SVD method [8].

We establish a complete rotation matrix with an assumption that there still exist some errors to compensate for as,

$$
R=R_{X} R_{Y} R_{Z}=R_{S V D_{x}} R_{\theta_{x}} R_{S V D_{y}} R_{\theta_{y}} R_{S V D_{z}} R_{\theta_{z}}
$$

Where $R: 3 \times 3$ rotation matrix , $R_{X}=R_{S V D_{x}} R_{\theta_{x}}, R_{S V D_{x}}, R_{S V D_{y}}, R_{S V D_{z}}$ : Rotation matrix obtained from the SVD, $R_{\theta_{x}}, R_{\theta_{Y}}, R_{\theta_{Z}}$ : Error rotation matrix. Since the inverse of the complete rotation matrix must be an input rotated face of frontal view,

$$
\mathbf{p}_{\mathbf{i}}=R^{-1} \mathbf{p}_{\mathbf{i}}^{\prime}=R_{Z}^{-1} R_{Y}^{-l} R_{X}^{-1} \mathbf{p}_{\mathbf{i}}^{\prime}=R_{\theta_{z}}^{-l} R_{S V D_{z}}^{-l} R_{\theta_{y}}^{-1} R_{S V D_{y}}^{-l} R_{\theta_{x}}^{-1} R_{S V D_{x}}^{-l} \mathbf{p}_{\mathbf{i}}^{\prime}
$$

where $\mathrm{p}_{\mathbf{i}}^{\prime}, \mathrm{p}_{\mathbf{i}}$ are feature vectors before and after rotation.

After rotating the estimated angle obtained from the SVD method about the X axis, the error $\theta_{x}$ is supposed to be computed for compensating. To estimate $\theta_{x}$, we exploit the $\mathrm{X}$ axis rotation matrix for evaluation. The key feature point is the NPP because all the NPPs of the 3D face model and the input are normalized to the fixed point $p(0,0, z)$ when the face is frontal. We can estimate $\theta_{x}$ from the follow equation.

$$
\begin{gathered}
\mathbf{p}^{\prime}=R_{X}^{-1} \mathbf{n}=R_{\theta_{x}}^{-1} R_{S V D_{x}}^{-1} \mathbf{n} \\
\therefore \theta_{x}=\arctan \left(\frac{y \cos \theta_{S V D_{x}}-z \sin \theta_{S V D_{x}}}{y \sin \theta_{S V D_{x}}+z \cos \theta_{S V D_{x}}}\right)
\end{gathered}
$$

The similar refinement procedure is applied to estimate the error $\theta_{y}$.

$$
\therefore \theta_{y}=\arctan \left(\frac{x \cos \theta_{S V D_{y}}-z^{\prime} \sin \theta_{S V D_{y}}}{x \sin \theta_{S V D_{y}}+z^{\prime} \cos \theta_{S V D_{y}}}\right)
$$

The error angle for $\theta_{z}$ can be obtained from the method in [8]. When the face vector is denoted as $\vec{F}(a, b, c)$, which is a vertical vector connected from the minimum point of the nose ridge to the center point of the left and right eyebrow.

$$
\theta_{z}=\arcsin \left(\frac{-a}{\sqrt{a^{2}+b^{2}+c^{2}}}\right)
$$




\section{Face Recognition}

In this section, we present a novel face recognition method using the face curvature shape indexes with dynamic programming. Fig. 2 describes the proposed procedure for face recognition. We extract feature points which are defined as areas with large shape variation measured by shape index calculated from principal curvatures [9].

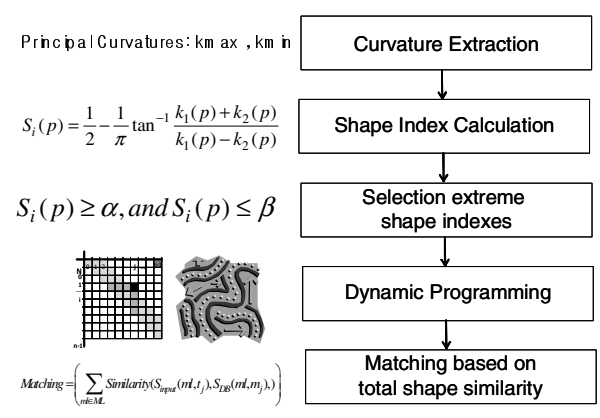

Fig. 2. The proposed face recognition procedure

Shape index $S_{i}(p)$, a quantitative measure of the shape of a surface point $p$, is

$$
S_{i}(p)=\frac{1}{2}-\frac{1}{\pi} \tan ^{-1} \frac{k_{1}(p)+k_{2}(p)}{k_{1}(p)-k_{2}(p)}
$$

Where $k_{1}(p)$ and $k_{2}(p)$ are maximum and minimum principal curvatures. These shape indexes are in the range of $[0,1]$. As we can see from [10], there are nine well-known shape categories and their locations on the shape index scale. Among those shape indexes, we select the extreme concave and convex points of curvatures as feature points. These feature points are distinctive for recognizing faces. Therefore, we select those shape indexes as feature points, feature ${ }_{i}(p)$, if a shape index $S_{i}(p)$ satisfies the following condition.

$$
\text { feature }_{i}(p)=\left\{\begin{array}{cc}
\partial \leq S_{i}(p)<1, & \text { concavity } \\
0<S_{i}(p) \leq \beta, & \text { convexity }
\end{array}\right.
$$

where $0<\partial, \beta<1$. With these selected facial shape indexes, we perform a dynamic programming in order to recognize the faces in the database [11].

We define a similarity measure and Total Shape Similarity Score (TSSS) as follow.

$$
\begin{gathered}
\operatorname{Similarity}\left(S_{\text {input }}, S_{D B}\right)=1-\mid \text { feature }_{\text {input }}-\text { feature }_{D B} \mid \\
T S S S=\sum_{n} \operatorname{Similarity}\left(S_{\text {input }}\left(i, c_{j}\right), S_{D B}\left(i, c_{j}, n\right),\right)
\end{gathered}
$$


where $S$ is denoted as facial shape index. $C_{j}$ is a face curvature and $\mathrm{n}$ is the number faces in the database. Score is a summation of the individual similarity score for each pair of matching descriptors.

\section{Experimental Results}

We test the 3D head pose estimation based on the proposed EC-SVD and face recognition rate under pose varying environments by using two different $3 \mathrm{D}$ sensors.

To evaluate the proposed EC-SVD algorithm, we first extract six facial feature points based on the geometrical configuration, Fig. 3 shows range images of the selected facial feature points of frontal (top row), left (middle row) and right (bottom row) pose variations. To estimate the head pose of an input data, we test range data on various rotation angles. The results are tabulated in Table 1 . We obtain various head poses of individuals and we acquire 7 head poses per person such as frontal, \pm 15 and \pm 30 for the $\mathrm{Y}$ axis, \pm 15 for the $\mathrm{X}$ axis as probe images.

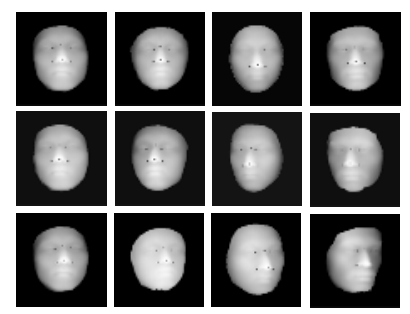

Fig. 3. 3D facial feature extraction for head pose estimation: Top row(frontal), second row(right pose) and third row(left pose)

Table 1. Mean absolute rotation error (degree) and translational error for each axis

\begin{tabular}{|c|c|c|c|c|c|c|c|}
\hline \multirow{2}{*}{ Test Images } & \multicolumn{3}{|c|}{$\begin{array}{c}\text { Mean Absolute Error using } \\
\text { SVD(Degree) }\end{array}$} & \multicolumn{3}{c|}{$\begin{array}{c}\text { Mean Absolute Error using } \\
\text { EC-SVD (Degree) }\end{array}$} & $\begin{array}{c}\text { Average } \\
\text { Translation } \\
\text { errors } \\
\text { (RMSE) }\end{array}$ \\
\cline { 2 - 7 } & X axis & Y axis & Z axis & X axis & Y axis & Z axis & 2.756 \\
\hline Face01 & 3.0215 & 4.3265 & 5.0124 & 0.8738 & 1.0125 & 1.5923 & 2.0083 \\
\hline Face14 & 3.0214 & 3.5216 & 5.1579 & 1.8991 & 0.9236 & 2.0080 & 2.457 \\
\hline Face23 & 2.3549 & 3.6546 & 3.0646 & 0.8680 & 1.1532 & 1.3783 & 3.175 \\
\hline $\begin{array}{c}\text { Average for } \\
\text { all faces }\end{array}$ & $\mathbf{2 . 8 7 6 5}$ & $\mathbf{3 . 6 5 6 3}$ & $\mathbf{3 . 8 5 6 5}$ & $\mathbf{0 . 8 5 6 5}$ & $\mathbf{0 . 9 6 5 4}$ & $\mathbf{1 . 5 2 1 2}$ & $\mathbf{2 . 6 1 4}$ \\
\hline
\end{tabular}

From the results shown in Table 1, we can confirm that the EC-SVD algorithm provides an estimated head pose for a different range of head poses. The error angle for each axis is compensated for any head poses when we normalize the NPP to the fixed point on the $\mathrm{Z}$ axis. Less than 1.6 degree error is resulted from our test results for each $\mathrm{X}, \mathrm{Y}$ and $\mathrm{Z}$ axis and it is highly acceptable for pose invariant face recognition. The proposed EC-SVD algorithm recovers the error angle remained by the SVD method, and it can be efficiently applied to pose invariant face. 
For the identification of a pose estimated range image, we compare the proposed method with the correlation matching and 3D Principal Component Analysis (PCA) [12]. For the proposed method, we first perform surface reconstruction of the faces from range images. We acquire very smooth facial surfaces from the $3 \mathrm{D}$ faces in the database, but discrete lines are appeared on the input face due to structured light patterns. Therefore, we extract curvatures which should be distinctive features for individuals, and adopt this feature which can be utilized for face recognition.

We extract 20 curvatures from the nose peak point which is in the center curvature. We select them based on sampling by two pixels towards the horizontal direction. Among them, we select facial shape indexes based on the threshold as mentioned in

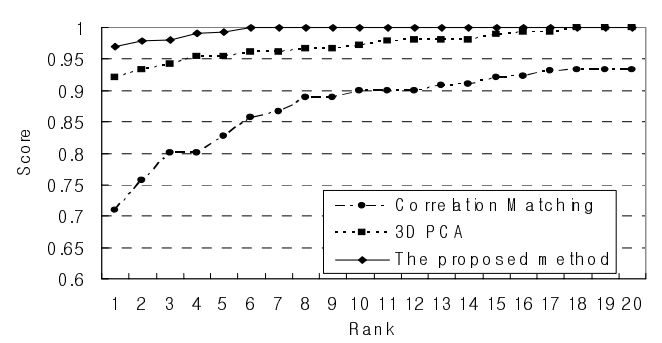

Fig. 4. Comparison of the face recognition rates under different poses

section 4 . The determined threshold value $\alpha$ for concave points is 0.75 , and $\beta$ is 0.25 for convex points. These values are selected based on the nine well-known shapes. We compare facial curvatures based on facial shape indexes based on dynamic programming for various head poses.

To describe the face matching, we tabulated matching results based on DP with facial shape indexes. When an input face is selected, we compare all the faces in the database based on the sum of facial shape indexes with DP, finally get a Total Shape Similarity Score (TSSS) for matching. From the experimental results, even though we get the less number of shape indexes than some faces, the TSSS of the identical face in the database is the highest among them. That is, facial shape indexes are distinctive features for face recognition. As we can see from Fig. 4, we have higher recognition rate according to the proposed method. We have $72 \%$ recognition rate for the correlation matching and $92 \%$ at first rank by the 3D PCA. However, we obtain $96.8 \%$ based on the proposed method at first rank under seven different poses. From the simulation results, we have effectively utilized facial shape indexes for pose invariant face recognition and achieved satisfactory recognition rate based on the proposed method.

\section{Conclusion}

In this paper, we proposed the face recognition method based on facial shape indexes by using two different 3D sensors under pose varying environments. We utilized the advantages of each 3D sensor such as real time 3D data acquisition system for the input and high quality images of $3 \mathrm{D}$ heads for the database. 
As we can see from the results, we obtained accurate 3D head pose estimation results using the EC-SVD procedure, and the final estimation errors of the 3D head pose estimation in our proposed method were less than 1.6 degree on average for each axis. In addition, our 3D facial feature extraction is automatically performed and assured that geometrically extracted feature points were efficient to estimate the head pose. For face recognition, we used facial shape indexes for recognizing faces with dynamic programming. We obtained $96.8 \%$ face recognition rate at first rank based on the proposed method which is highly acceptable results for pose invariant face recognition. We are now researching expression invariant face recognition with more $3 \mathrm{D}$ faces.

\section{Acknowledgments}

This work was supported by the Korea Science and Engineering Foundation (KOSEF) through the Biometrics Engineering Research Center (BERC) at Yonsei University.

\section{References}

1. R. Chellappa, C. L Wilson, and S. Sirohey, "Human and machine recognition of faces : A survey," Proceedings of the IEEE, vol. 83, pp. 705-740, May 1995.

2. W. Zhao, R. Chellappa, P. J. Phillips, and A. Rosenfeld, "Face recognition: A literature survey," ACM, Computing Surveys, Vol. 35, No.4, Dec. 2003.

3. H. T. Tanaka, M. Ikeda and H. Chiaki, "Curvature-based face surface recognition using spherical correlation," Proceedings of the Third International Conference on Automatic Face and Gesture Recognition, pp.372-377, 1998.

4. C. S. Chua, F. Han, and Y. K. Ho, "3D human face recognition using point signature," Proc. of the Fourth International Conference on Automatic Face and Gesture Recognition, pp.233-238, 2000.

5. C. Hesher, A. Srivastava, and G. Erlebacher, "A novel technique for face recognition using range images," Proceedings of the Seventh Int'l Symp. on Signal Processing and Its Applications, 2003.

6. G. Medioni and R. Waupotitsch, "Face recognition and modeling in 3D," Proceedings of the IEEE Int'l Workshop on Analysis and Modeling of Faces and Gestures (AMFG 2003), pp. 232-233, 2003.

7. T.S. Huang, A.N. Netravali, "Motion and structure from feature correspondences: A Review," Proceedings of the IEEE, vol. 82, no. 2, pp. 252-268, 1994.

8. H. Song, J. Kim, S. Lee and K. Sohn, “3D sensor based face recognition,” Applied Optics, vol. 44, No. 5, pp.677-687, Feb. 2005.

9. G. G. Gordon, "Face recognition based on depth maps and surface curvature," SPIE Proceedings : Geometric Methods in Computer Vision, San Diego, CA, Proc. SPIE 1570, 1991.

10. C. Dorai and A. K. Jain, "COSMOS-A Representation Scheme for 3D Free-Form Objects," IEEE Trans. on Pattern Anal. and Machine Intell., vol. 19, no. 10, pp. 1115-1130, Oct. 1997.

11. D. P. Bertsekas, Dynamic Programming and Optimal Control : $2^{\text {nd }}$ Edition, ISBNs : 1 886529-09-4, Nov. 2000.

12. K. Chang, K. Bowyer, and P. Flynn, "Face recognition using $2 \mathrm{D}$ and 3D facial data," Proceeding of the Multimodal User Authentication Workshop, pp 25-32, 2003. 